\title{
Friction-Induced Velocity Fields for Point Parts Sliding on a Rigid Oscillated Plate
}

\author{
Thomas H. Vose, Paul Umbanhowar, and Kevin M. Lynch
}

\begin{abstract}
We show that small-amplitude periodic motion of a rigid plate causes point parts on the plate to move as if they are in a position-dependent velocity field. Further, we prove that every periodic plate motion maps to a unique velocity field. By allowing a plate to oscillate with six-degrees-of-freedom, we can create a large family of programmable velocity fields. We examine in detail sinusoidal plate motions that generate fields with either isolated sinks or squeeze lines. These fields can be exploited to perform tasks such as sensorless part orientation.
\end{abstract}

\section{INTRODUCTION}

When parts are in contact with a rigid oscillating surface, frictional forces induce the parts to move in a predictable manner. A single rigid plate is therefore a simple and appealing platform on which to perform a variety of parts manipulation tasks such as transporting, orienting, positioning, sorting, mating, etc. Because the motion of the plate is programmable, it is possible to perform these tasks on parts of various shapes and sizes without the need to reconfigure hardware for each new task or part geometry.

In this paper we propose a general model of part motion for point parts on a six-degree-of-freedom (DoF) oscillating plate. The key discovery is that small-amplitude periodic plate motions map to position-dependent velocity fields on the plate surface, which we refer to as asymptotic velocity fields. For many plate motions and coefficients of friction, part motion is well described by the asymptotic velocity field. Although we do not yet know how to characterize the set of all asymptotic velocity fields obtainable with a six-DoF oscillating plate, we do know that it includes fields with nonzero divergence (i.e., fields with sinks and sources). The video accompanying this paper shows parts on our six-DoF prototype device undergoing motion in such fields.

The rest of the paper is laid out as follows: in Section II we discuss related work that focuses on programmable force fields and vibratory surfaces; in Section III we present a full dynamic model of the part-plate system; in Section IV we present a simplified dynamic model which is the basis for our theorem asserting the existence of asymptotic velocity fields; in Sections V and VI we explain how to estimate asymptotic velocity fields and offer examples for sinusoidal plate motions; and in Section VII we conclude with remarks on future work.

T. H. Vose, P. Umbanhowar, and K. M. Lynch are with the Department of Mechanical Engineering, Northwestern University, Evanston, IL 60208, USA (e-mail: $\{t$-vose, umbanhowar, kmlynch\}@northwestern.edu)

This work was supported by NSF grants IIS-0308224 and CMMI-0700537 and Thomas Vose's NSF fellowship.

\section{BACKGROUND}

For some tasks, such as positioning and orienting parts, planar force fields with nonzero divergence (e.g., squeeze fields and sink fields) can be designed to interact with the part so that the task can be completed without the use of sensors. Significant theoretical work has gone into developing algorithms that exploit programmable force fields for sensorless positioning and orienting of planar parts (e.g., [1], [2], [3], [4]). Most devices designed to generate programmable force fields do so using a planar array of actuators, such as MEMS elements [5], [6], [7], rolling wheels [8], air jets [9], or vibrating plates [10]. Although these systems can create a wide range of fields, the fields are necessarily discrete, whereas most of the theoretical work assumes continuity. To approximate a continuous field, the array must contain a large number of actuators, often making fabrication and control difficult. Our current prototype vibratory device, which consists of just a single rigid plate and six voice coil actuators that allow for sixDoF motion of the plate, is comparatively simple yet powerful enough to create continuous fields with nonzero divergence.

Our work is a natural extension of the Universal Planar Manipulator (UPM) designed by Canny and Reznik [11]. The UPM consists of a single rigid horizontal plate that moves with three degrees-of-freedom (two translational and one rotational). Systems that restrict the plate motion to the horizontal plane, such as the UPM, can only generate frictional force fields with zero divergence [12]. Therefore, position sensing is required to orient and position parts. What allows our system to create fields with nonzero divergence is the plate's ability to move with all six degrees of freedom. In particular, the plate's ability to rotate out of the horizontal plane while simultaneously translating in the horizontal plane allows us to generate fields with sinks and sources.

The relationship between a periodic plate motion and the resultant frictional force acting on a planar part is generally quite complicated. However, when the plate motion is purely translational, point parts at all locations experience the same forces. This results in a single feed rate that is independent of position. In the simplest one-DoF case, the plate is horizontal and translates longitudinally. Reznik and Canny examined a particular type of bang-bang motion for this case in [13] and [14]. Okabe et al. looked at one-DoF plate motion in which the plate is also angled with respect to the horizontal [15]. Two-DoF systems, allowing translation both longitudinally and normal to the surface, are examined in [16] and [10]. In [17], Lynch and Umbanhowar derived optimal plate motions that maximize part speed on one- and two-DoF translating 
rigid plates. The three-DoF UPM is capable of generating certain position-dependent force fields [12] including localized ones [18].

In [19] we first demonstrated that fields with nonzero divergence can be created with a six-DoF rigid plate that oscillates periodically. This paper extends that work in three ways: it formally defines asymptotic velocity, it proves that every periodic plate motion maps to a unique asymptotic velocity field, and it introduces new plate motions that simplify the way some of the fields in [19] can be generated.

\section{SySTEM MODEL}

\section{A. Plate Kinematics}

Consider a rigid plate undergoing small-amplitude vibration. We define three coordinate systems: a fixed inertial frame $\mathcal{W}$, a local frame $\mathcal{S}$ attached to the origin of the plate, and an inertial frame $\mathcal{S}^{\prime}$ instantaneously aligned with $\mathcal{S}$ (Figure 1). The $z$-axis of $\mathcal{W}$ is in the direction opposite the gravity vector, which is represented as $\mathbf{g}=[0,0,-g]^{T}$ in the $\mathcal{W}$ frame. The $z_{\mathcal{S}}$-axis of $\mathcal{S}$ is perpendicular to the plate surface.

We choose to describe the kinematics of the plate in the $\mathcal{W}$ frame. The configuration of the plate is given by

$$
\left[\begin{array}{cc}
\mathbf{R} & \mathbf{p} \\
0 & 1
\end{array}\right] \in S E(3)
$$

where $\mathbf{R} \in S O(3)$. Both $\mathbf{R}$ and $\mathbf{p}$ are periodic $C^{1}$ functions of time with period $T$. In the home position, $\mathbf{p}=\mathbf{0}$ and $\mathbf{R}=\mathbf{I}$, where $\mathbf{I}$ is the identity matrix. The linear velocity of the origin of the plate is $\dot{\mathbf{p}}$ and the angular velocity of the plate is $\boldsymbol{\omega}$. The linear acceleration of the origin of the plate is $\ddot{\mathbf{p}}=\left[\ddot{p}_{x}, \ddot{p}_{y}, \ddot{p}_{z}\right]^{T}$ and the angular acceleration of the plate is $\boldsymbol{\alpha}=\left[\alpha_{x}, \alpha_{y}, \alpha_{z}\right]^{T}$. In general, we choose to specify the plate's motion in terms of $\ddot{\mathbf{p}}$ and $\boldsymbol{\alpha}$.

\section{B. Part Kinematics}

Let $\mathrm{P}$ be a point part with mass $m$ in contact with the plate. As illustrated in Figure 1, let $\mathbf{q}=\left[x_{\mathcal{S}}, y_{\mathcal{S}}, 0\right]^{T}$ be a vector in $\mathcal{S}$ to $\mathrm{P}$, and $\mathbf{r}=[x, y, z]^{T}$ be a vector in $\mathcal{W}$ to $\mathrm{P}$ such that

$$
\mathbf{r}=\mathbf{p}+\mathbf{R q} .
$$

Let $\mathrm{P}^{*}$ be the point on the plate directly underneath $\mathrm{P}$. The position of $\mathrm{P}^{*}$ is given by the vector $\mathbf{r}^{*}$ in the $\mathcal{W}$ frame. The velocity and acceleration of $\mathrm{P}^{*}$ in the $\mathcal{W}$ frame are given by

$$
\begin{aligned}
& \dot{\mathbf{r}}^{*}=\dot{\mathbf{p}}+\boldsymbol{\omega} \times \mathbf{R q} \\
& \ddot{\mathbf{r}}^{*}=\ddot{\mathbf{p}}+\boldsymbol{\omega} \times \boldsymbol{\omega} \times \mathbf{R q}+\boldsymbol{\alpha} \times \mathbf{R q} .
\end{aligned}
$$

The velocity and acceleration of $\mathrm{P}$ in the $\mathcal{W}$ frame are given by

$$
\begin{aligned}
\dot{\mathbf{r}} & =\dot{\mathbf{p}}+\boldsymbol{\omega} \times \mathbf{R} \mathbf{q}+\mathbf{R} \dot{\mathbf{q}} \\
& =\dot{\mathbf{r}}^{*}+\mathbf{R} \dot{\mathbf{q}} \\
\ddot{\mathbf{r}} & =\ddot{\mathbf{p}}+\boldsymbol{\omega} \times \boldsymbol{\omega} \times \mathbf{R} \mathbf{q}+\boldsymbol{\alpha} \times \mathbf{R q}+2 \boldsymbol{\omega} \times \mathbf{R} \dot{\mathbf{q}}+\mathbf{R} \ddot{\mathbf{q}} \\
& =\ddot{\mathbf{r}}^{*}+2 \boldsymbol{\omega} \times \mathbf{R} \dot{\mathbf{q}}+\mathbf{R} \ddot{\mathbf{q}} .
\end{aligned}
$$

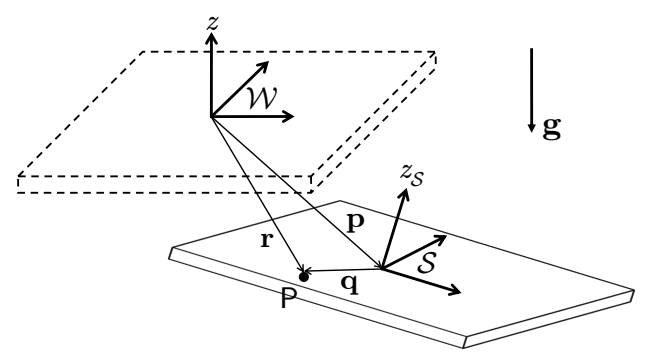

Fig. 1. An extremely exaggerated picture of the plate displaced from the fixed $\mathcal{W}$ frame by the vector $\mathbf{p}$. The position of the part $\mathrm{P}$ is given by $\mathbf{r}$ in the $\mathcal{W}$ frame and by $\mathbf{q}$ in $\mathcal{S}$ frame.

\section{Part Dynamics}

Three forces act on the part: gravity, friction, and the normal force from the plate (Figure 2). Applying Newton's second law in the $\mathcal{S}^{\prime}$ frame gives

$$
\begin{aligned}
\mathbf{f}_{\mathrm{N}_{\mathcal{S}^{\prime}}}+\mathbf{f}_{\mathrm{F}_{\mathcal{S}^{\prime}}}+\mathbf{f}_{\mathrm{G}_{\mathcal{S}^{\prime}}} & =m \mathbf{R}^{T} \ddot{\mathbf{r}} \\
& =m \mathbf{R}^{T}\left(\ddot{\mathbf{r}}^{*}+2 \boldsymbol{\omega} \times \mathbf{R} \dot{\mathbf{q}}\right)+m \ddot{\mathbf{q}},
\end{aligned}
$$

where $\mathbf{f}_{\mathrm{N}_{\mathcal{S}^{\prime}}}=[0,0, N]^{T}, \mathbf{f}_{\mathrm{F}_{\mathcal{S}^{\prime}}}=\left[F_{x_{\mathcal{S}}}, F_{y_{\mathcal{S}}}, 0\right]^{T}$, and $\mathbf{f}_{\mathrm{G}_{\mathcal{S}^{\prime}}}=$ $m \mathbf{R}^{T} \mathbf{g}$ are the normal, frictional, and gravitational forces on the part in the $\mathcal{S}^{\prime}$ frame. Solving (6) for $\ddot{\mathbf{r}}$ yields an expression for the part's acceleration in the $\mathcal{W}$ frame:

$$
\ddot{\mathbf{r}}=\frac{1}{m} \mathbf{R}\left(\mathbf{f}_{\mathrm{N}_{\mathcal{S}^{\prime}}}+\mathbf{f}_{\mathrm{F}_{\mathcal{S}^{\prime}}}\right)+\mathbf{g} .
$$

Explicit expressions for $\mathbf{f}_{\mathrm{N}_{\mathcal{S}^{\prime}}}$ and $\mathbf{f}_{\mathrm{F}_{\mathcal{S}^{\prime}}}$ are derived in the following two sections.

The state vector $\mathbf{x}_{\mathcal{W}}=[\mathbf{r}, \dot{\mathbf{r}}]^{T}$ can be computed by integrating (8). The state vector $\mathbf{x}_{\mathcal{S}}=[\mathbf{q}, \dot{\mathbf{q}}]^{T}$ can be computed from $\mathbf{x}_{\mathcal{W}}$ noting that (1) and (4) imply

$$
\begin{aligned}
\mathbf{q} & =\mathbf{R}^{T}(\mathbf{r}-\mathbf{p}) \\
\dot{\mathbf{q}} & =\mathbf{R}^{T}\left(\dot{\mathbf{r}}-\dot{\mathbf{r}}^{*}\right) .
\end{aligned}
$$

Our analysis is restricted to situations in which the part always remains in contact with the plate. Contact is maintained as long as the magnitude of the normal force is positive. Additionally, contact implies the acceleration of the part perpendicular to the plate surface is zero at all times in the $\mathcal{S}$ frame. Mathematically, we express this as

$$
\mathbf{z}^{T} \ddot{\mathbf{q}}=0,
$$

where $\mathbf{z} \triangleq[0,0,1]^{T}$.

\section{Normal Force}

As noted previously, the normal force has the form $\mathbf{f}_{\mathrm{N}_{\mathcal{S}^{\prime}}}=$ $[0,0, N]^{T}$ in the $\mathcal{S}^{\prime}$ frame. Pre-multiplying (7) by $\mathbf{z}^{T}$ and noting (11) yields the following expression for the magnitude of the normal force, $N$ :

$$
N=m \mathbf{z}^{T}\left[\mathbf{R}^{T}\left(\ddot{\mathbf{r}}^{*}+2 \boldsymbol{\omega} \times \mathbf{R} \dot{\mathbf{q}}-\mathbf{g}\right)\right] .
$$

We define the effective gravity as

$$
g_{\mathrm{eff}}=\mathbf{z}^{T}\left[\mathbf{R}^{T}\left(\ddot{\mathbf{r}}^{*}+2 \boldsymbol{\omega} \times \mathbf{R} \dot{\mathbf{q}}-\mathbf{g}\right)\right],
$$

so that $N=m g_{\text {eff }}$. 


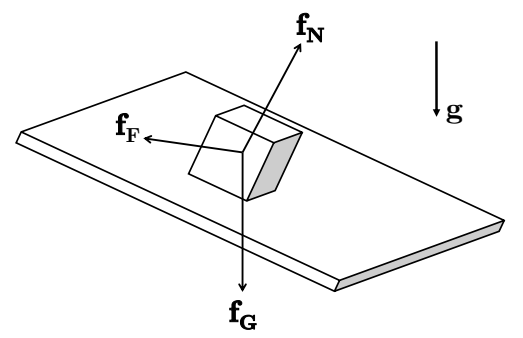

Fig. 2. The three forces that act on the part are due to gravity, friction, and the normal force from the plate. The gravitational force $\mathbf{f}_{G}$ always acts in the negative $z$-direction of the $\mathcal{W}$ frame, the frictional force $\mathbf{f}_{F}$ always acts tangent to the plate surface, and the normal force $\mathbf{f}_{N}$ always acts perpendicular to the plate surface.

\section{E. Frictional Force}

We assume Coulomb friction in our model. Since frictional forces can only act in the $x-y$ plane of the $\mathcal{S}^{\prime}$ frame we define the matrix $\mathbf{S}_{x y}$ that projects vectors in $\mathbb{R}^{3}$ onto the $x-y$ plane.

The frictional force acting on a part located at $\mathbf{r}$ depends on the state of the system. There are three cases, which we summarize mathematically as:

$$
\mathbf{f}_{\mathrm{F}_{\mathcal{S}^{\prime}}}= \begin{cases}-\mu_{k} N \frac{\dot{\mathbf{q}}}{\|\dot{\mathbf{q}}\|}, & \|\dot{\mathbf{q}}\|>0 \\ \mu_{s} N \frac{\mathbf{S}_{x y} \mathbf{R}^{T} \ddot{\mathbf{r}}^{*}}{\left\|\mathbf{S}_{x y} \mathbf{R}^{T} \ddot{\mathbf{r}}^{*}\right\|}, & \|\dot{\mathbf{q}}\|=0 \\ & \left\|\mathbf{S}_{x y} \mathbf{R}^{T} \ddot{\mathbf{r}}^{*}\right\|>\mu_{s} g_{\mathrm{eff}} ; \\ m \mathbf{S}_{x y} \mathbf{R}^{T} \ddot{\mathbf{r}}^{*}, & \|\dot{\mathbf{q}}\|=0 \\ & \left\|\mathbf{S}_{x y} \mathbf{R}^{T} \ddot{\mathbf{r}}^{*}\right\| \leq \mu_{s} g_{\mathrm{eff}} ;\end{cases}
$$

where $\mu_{k}$ and $\mu_{s}$ are the respective kinetic and static coefficients of friction between the part and the plate.

\section{Mapping Periodic Plate Motions to POSITION-DEPENDENT PART VELOCITIES}

It is usually difficult to gain conceptual insight into the relationship between plate motion and part motion using the full dynamic model presented in the previous section. However, by running numerical simulations of the system, we observed that there is a unique average velocity $\mathbf{v}_{\mathrm{a}}(\mathbf{r})$ such that a point part at $\mathbf{r}$, moving with any other average velocity (when averaged over one cycle), tends toward $\mathbf{v}_{\mathrm{a}}(\mathbf{r})$. We call $\mathbf{v}_{\mathrm{a}}(\mathbf{r})$ the asymptotic velocity at $\mathbf{r}$. Thus, a part's motion on the plate is given approximately by the position-dependent asymptotic velocity field, where the quality of the approximation depends on the rate of convergence to the asymptotic velocity at each location.

The simplified system model presented in this section allows us to justify the position-dependent asymptotic velocity observed in the simulations and leads to further insight about part motion induced by small-amplitude periodic plate motions.

\section{A. Simplified System Model}

To simplify the system model, let us assume that the part is sliding at all times. Let us also operate in a regime where the period, linear displacement, and angular displacement of the plate are small enough so that we may assume $\mathbf{p} \approx \mathbf{0}$ and $\mathbf{R} \approx \mathbf{I}$. It follows that the part's position vector in the $\mathcal{W}$ frame can be approximated as $\mathbf{r} \approx \mathbf{q}=[x, y, 0]^{T}$, and that the gravitational, frictional, and normal forces acting on the part can be considered aligned with the $\mathcal{W}$ axes. In other words, the configuration of the plate is assumed to correspond to the home position at all times.

With the assumptions above, the approximate acceleration of the part in the horizontal plane, denoted by $\mathbf{a}$, is obtained by simplifying the $x$ and $y$ components of (8):

$$
\mathbf{a}=\left[\begin{array}{c}
\ddot{x} \\
\ddot{y} \\
0
\end{array}\right]=S_{x y} \ddot{\mathbf{r}} \approx-\mu_{k} g_{\mathrm{eff}} \frac{\dot{\mathbf{q}}}{\|\dot{\mathbf{q}}\|} .
$$

The effective gravity $g_{\text {eff }}$ and the relative velocity vector $\dot{\mathbf{q}}$ that respectively dictate the magnitude and direction of a can be approximated by simplifying (13) and (10) and further assuming that Coriolis and centripetal accelerations are insignificant:

$$
\begin{aligned}
g_{\mathrm{eff}} & \approx \mathbf{z}^{T}(\ddot{\mathbf{p}}+\boldsymbol{\alpha} \times \mathbf{r}-\mathbf{g}) \\
\dot{\mathbf{q}} & \approx \mathbf{S}_{x y}\left(\dot{\mathbf{r}}-\dot{\mathbf{r}}^{*}\right) .
\end{aligned}
$$

In summary, the simplified system model assumes the part is always sliding, the configuration of the plate always corresponds to the home position, and Coriolis and centripetal accelerations are negligible.

\section{B. Asymptotic Velocity of Sliding Parts}

Let $\mathbf{v}$ and $\mathbf{v}^{*}$ be the respective velocities of the part and the plate at a location $\mathbf{r}$ projected onto the $x-y$ velocity plane in the $\mathcal{W}$ frame. We refer to this plane of velocities as $\mathcal{V}_{x y}$.

Let $\mathbf{r}_{x y}=[x, y]^{T}$. At a given $\mathbf{r}_{x y}, \mathbf{v}^{*}$ sweeps out a closed trajectory in $\mathcal{V}_{x y}$ for all periodic plate motions. Let $\mathcal{C H}^{*}$ denote the convex hull of this trajectory in the $\mathcal{V}_{x y}$ plane.

Even if the part has a nonzero velocity, we assume its displacement is negligible during a cycle of plate motion. Let us therefore denote by $\Sigma$ a system consisting of

1) a plate undergoing periodic motion;

2) a part with a fixed value of $\mathbf{r}_{x y}$, but with a velocity that may be nonzero, and an acceleration given by (15) of the simplified dynamic model.

The following theorem asserts the existence of a unique asymptotic velocity for this type of system.

Theorem 1: For a system $\Sigma$, the part asymptotically converges from any initial velocity to a unique stable limit cycle of period $T$ on or inside $\mathcal{C H}^{*}$.

Sketch of Proof: We first show that the part's velocity converges asymptotically to $\mathcal{C H}^{*}$ in the $\mathcal{V}_{x y}$ plane. From (15) and (17), the part accelerates such that $\mathbf{v}$ moves in the direction of $\mathbf{v}^{*}$ at each instant in the $\mathcal{V}_{x y}$ plane. Recalling that $\mathbf{v}^{*}$ sweeps out a closed trajectory, it follows that if $\mathbf{v}$ is outside of $\mathrm{CH}^{*}$ it must always move closer to $\mathcal{C H}^{*}$, and if $\mathbf{v}$ is contained in $\mathcal{C H}^{*}$ it can never escape.

Now we show that the part's velocity converges to a unique stable limit cycle in $\mathcal{C H}^{*}$. Let $\mathrm{P}_{1}$ and $\mathrm{P}_{2}$ be two point parts located at $\mathbf{r}_{x y}$ with identical coefficients of kinetic friction, $\mu_{k}$. Let the velocities of $\mathrm{P}_{1}$ and $\mathrm{P}_{2}$ in the $\mathcal{V}_{x y}$ plane be $\mathbf{v}_{1}$ 
and $\mathbf{v}_{2}$. Let $\Delta v=\left\|\mathbf{v}_{1}-\mathbf{v}_{2}\right\|$ be the distance between $\mathbf{v}_{1}$ and $\mathbf{v}_{2}$ in the $\mathcal{V}_{x y}$ plane (Figure 3).

From (16) the value of $g_{\text {eff }}$ is the same for both $\mathrm{P}_{1}$ and $P_{2}$. Thus, (15) implies that at all times $P_{1}$ and $P_{2}$ accelerate with equal magnitude in the direction of $\mathbf{v}^{*}$ in the $\mathcal{V}_{x y}$ plane. It immediately follows that $\frac{d}{d t}(\Delta v) \leq 0$. The nondecreasing case $\left(\frac{d}{d t}(\Delta v)=0\right)$ corresponds to when $\mathbf{v}_{1}, \mathbf{v}_{2}$, and $\mathbf{v}^{*}$ are collinear such that $\mathbf{v}^{*}$ is not between $\mathbf{v}_{1}$ and $\mathbf{v}_{2}$. However, the periodic motion of the plate ensures that there will always be a duration of time $\tau>0$ during the cycle when $\mathbf{v}_{1}, \mathbf{v}_{2}$, and $\mathbf{v}^{*}$ are not collinear, or $\mathbf{v}_{1}, \mathbf{v}_{2}$, and $\mathbf{v}^{*}$ are collinear such that $\mathbf{v}^{*}$ is between $\mathbf{v}_{1}$ and $\mathbf{v}_{2}$. In either case, $\frac{d}{d t}(\Delta v)<0$ during this time, ensuring a contractive mapping over the course of a cycle. Thus, all parts located at $\mathbf{r}_{x y}$ must converge to a unique stable limit cycle on or inside $\mathcal{C H}^{*}$ that we call the asymptotic trajectory at $\mathbf{r}_{x y}$. The time-averaged velocity of the points on the asymptotic trajectory at $\mathbf{r}_{x y}$ is the unique asymptotic velocity $\mathbf{v}_{\mathbf{a}}\left(\mathbf{r}_{x y}\right)$.

Finally, we show that the period of the asymptotic trajectory must be the same as that of the plate. Consider an arbitrary periodic plate motion with period $T$. Assume that an asymptotic trajectory at $\mathbf{r}$ on or inside $\mathcal{C H}^{*}$ exists for this plate motion with period $T^{\prime}$ such that $T \neq T^{\prime}$.

For the case in which $T>T^{\prime}$ there must exist at least one point on the part's asymptotic trajectory that corresponds to at least two different points on the plate's trajectory in the $\mathcal{V}_{x y}$ plane. From (15) and (17), this implies that the part must accelerate in two different directions simultaneously, which is not physically possible.

For the case in which $T<T^{\prime}$ there must exist at least two points on the part's asymptotic trajectory that correspond to a single point on the plate's trajectory. Suppose that two parts, $\mathrm{P}_{1}$ and $\mathrm{P}_{2}$, have initial velocities in the $\mathcal{V}_{x y}$ plane corresponding to $\mathbf{v}_{1}$ and $\mathbf{v}_{2}$ at the instant when the plate has velocity $\mathbf{v}^{*}$. By definition, the velocities of $P_{1}$ and $P_{2}$ are once again $\mathbf{v}_{1}$ and $\mathbf{v}_{2}$ every $n T^{\prime}$ seconds, where $n=1,2,3, \ldots$. However, because $n$ can be chosen such that $n T^{\prime}>T$, this is a contradiction since the distance between $\mathbf{v}_{1}$ and $\mathbf{v}_{2}$ on or inside $\mathcal{C H}^{*}$ must decrease during any cycle of plate motion (i.e., any duration of $T$ seconds).

Figure 4 shows simulation results that illustrate the ideas presented in Theorem 1. The plots are for the location $\mathbf{r}_{x y}=$ $(0.06,0)^{T} \mathrm{~m}$ on a plate undergoing the motion described in Figure 5(k). In Figure 4(a), two parts with different initial velocities are shown converging to the same asymptotic trajectory in $\mathcal{C H}^{*}$. This is also highlighted in Figure 4(b), which shows $\Delta v$ decreasing over time, and in Figure 4(c), which shows the individual $x$ and $y$ velocities of the two parts converging in time. The markers in Figure 4(a) and (c) are plotted every half cycle to show that the asymptotic trajectory and the plate's trajectory have the same period.

\section{Computing Asymptotic Velocity}

Theorem 1 allows us to formally define the asymptotic velocity at $\mathbf{r}_{x y}$ as

$$
\mathbf{v}_{\mathrm{a}}\left(\mathbf{r}_{x y}\right)=\frac{1}{T} \int_{t}^{t+T} \mathbf{v}^{\prime}(t) d t,
$$

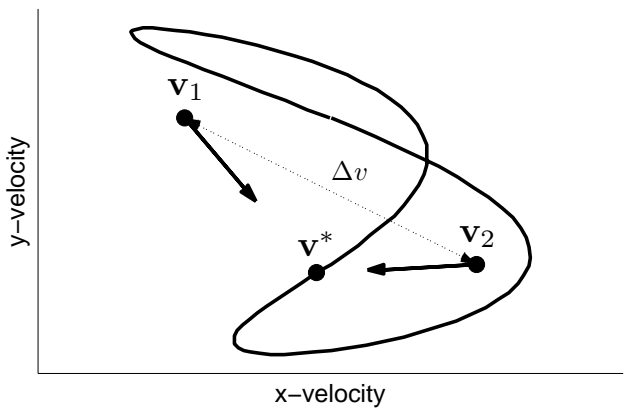

Fig. 3. The $\mathcal{V}_{x y}$ plane at an arbitrary location $\mathbf{r}$, in which the velocity of the plate $\mathbf{v}^{*}$ sweeps out a closed trajectory. From (15), the tangent to the trajectory of any point part (e.g., $\mathbf{v}_{1}$ ) passes through $\mathbf{v}^{*}$ at each instant.

where $\mathbf{v}^{\prime}(t)$ is the unique limit cycle from Theorem 1. For some simple plate motions, the asymptotic velocity field as a function of $\mathbf{r}_{x y}$ can be determined analytically from (18) (see e.g., [19], [17], [20]). Otherwise, it can be determined numerically by computing the asymptotic velocity at a discrete set of points on the plate as follows:

1) Set the part's initial velocity to zero.

2) Simulate the part dynamics (without updating the position) for one cycle of plate motion.

3) Subtract the part's velocity at the end of the cycle from its velocity at the beginning of the cycle. If the magnitude of the difference is not within a predefined tolerance $\epsilon$, repeat step 2, but use the part's velocity at the end of the current cycle as its initial velocity for the next cycle.

4) Average the part's velocity over the cycle.

\section{Estimating Asymptotic Velocity}

Although the asymptotic velocity can always be computed numerically, in this section we explain how to estimate it using the notion of transient acceleration. The advantage of this is that the transient acceleration is defined such that it is independent of the part's velocity and therefore does not require simulation to compute.

For some plate motions a qualitative estimate of the transient acceleration can be obtained by simple inspection. This is useful for gaining intuition about the properties of the fields generated by certain classes of plate motions, as discussed in Section VI. It also leads to insight about the inverse problem: finding a plate motion that approximately generates a desired field.

\section{A. Transient Acceleration}

Let a point part located at $\mathbf{r}_{x y}$ have an initial velocity $\mathbf{v}=\mathbf{0}$ in the $\mathcal{V}_{x y}$ plane. Unless the part happens to begin exactly on the asymptotic trajectory, there is a transient period during the first few cycles of plate motion in which the part converges to the asymptotic trajectory at $\mathbf{r}_{x y}$. During the transient period the average acceleration of the part is nonzero in order to bring the average cycle velocity closer to the asymptotic velocity. Thus, to a good approximation, the asymptotic velocity at $\mathbf{r}_{x y}$ is proportional to the average transient acceleration over a small 


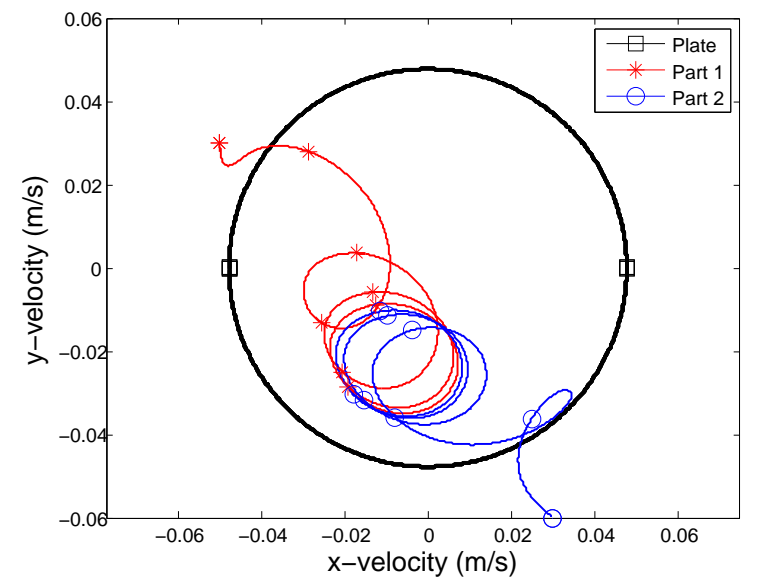

(a)

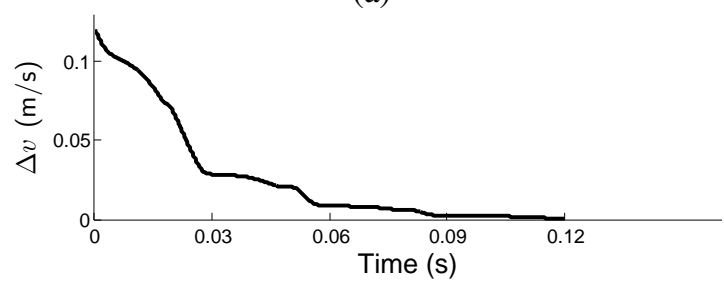

(b)

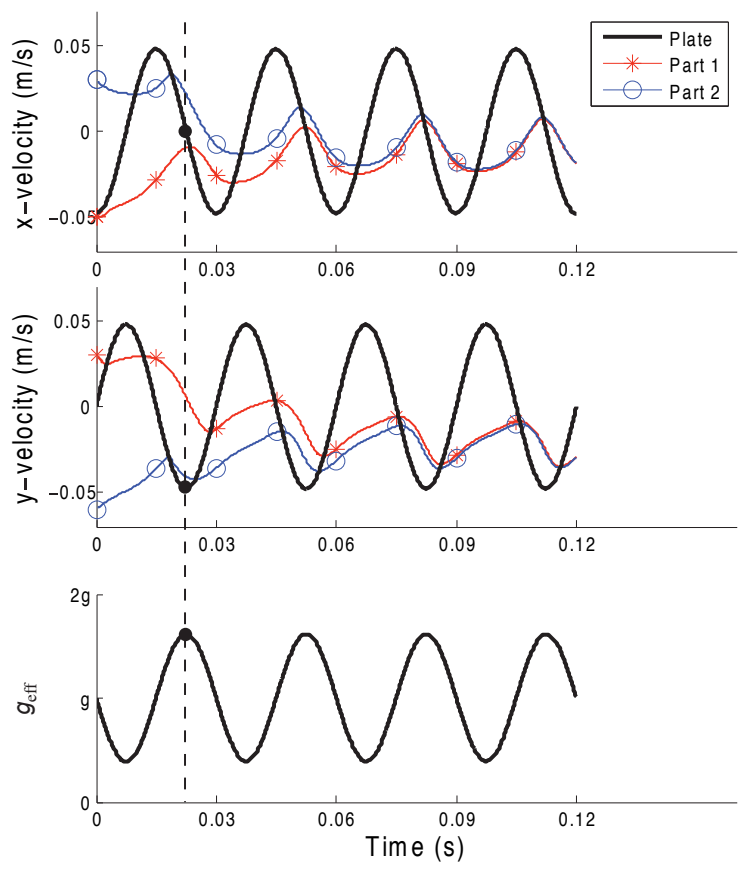

(c)

Fig. 4. A detailed look at two parts located at $(0.06,0) \mathrm{m}$ with different initial velocities on a plate undergoing the motion specified in Figure $5(\mathrm{k})$. The period of the plate's motion is $T=0.03 \mathrm{~s}$, and the markers in (a) and (c) are plotted every half cycle $(0.015 \mathrm{~s})$. In (a), the trajectory of the two parts in the $\mathcal{V}_{x y}$ plane is shown for four cycles of plate motion. Both parts rapidly approach a nearly circular asymptotic trajectory centered around $(-0.01,-0.025) \mathrm{m} / \mathrm{s}$ that is fully contained the convex hull of the plate's trajectory. The asymptotic trajectory and the plate's trajectory each have two markers per cycle indicating that they have the same period. The distance between the parts' velocities in the $\mathcal{V}_{x y}$ plane rapidly approaches zero without ever increasing, as shown in (b). The $x$ and $y$-components of the velocity are plotted individually vs. time in the top two graphs of (c); the effective gravity is plotted in the bottom graph of (c). The dashed vertical line in (c) denotes the time at which $g_{\text {eff }}$ is a maximum. number of cycles at $\mathbf{r}_{x y}$ arising from the initial condition $\mathbf{v}=$ 0 .

We use (15) to estimate the part's acceleration a during the transient. To estimate $g_{\text {eff }}$ we use (16), rewritten below explicitly in terms of acceleration components:

$$
g_{\mathrm{eff}} \approx \mathbf{z}^{T}(\ddot{\mathbf{p}}+\boldsymbol{\alpha} \times \mathbf{r}-\mathbf{g})=\ddot{p}_{z}+\alpha_{x} y-\alpha_{y} x+g .
$$

To estimate $\dot{\mathbf{q}}$ we assume that during the transient period the magnitude of the plate's velocity $\mathbf{v}$ is much greater than the part's velocity $\mathbf{v}^{*}$. Thus, (17) reduces to

$$
\dot{\mathbf{q}} \approx \mathbf{S}_{x y}\left(-\dot{\mathbf{r}}^{*}\right)=\mathbf{S}_{x y}(-\dot{\mathbf{p}}-\boldsymbol{\omega} \times \mathbf{r})=\left[\begin{array}{c}
-\dot{p}_{x}+\omega_{z} y \\
-\dot{p}_{y}-\omega_{z} x \\
0
\end{array}\right] .
$$

We make the important observation that in our model of the transient acceleration neither $g_{\text {eff }}$ nor $\dot{\mathbf{q}}$ depends on the motion of the part: from (19) the magnitude of a during the transient is a function of $\ddot{p}_{z}, \alpha_{x}$, and $\alpha_{y}$; from (20) the direction of a during the transient is a function of $\dot{p}_{x}, \dot{p}_{y}$, and $\omega_{z}$. We refer to $\ddot{p}_{z}, \alpha_{x}$, and $\alpha_{y}$ as the out-of-plane acceleration components of the plate's motion. We refer to $\dot{p}_{x}, \dot{p}_{y}$, and $\omega_{z}$ as the in-plane velocity components. In-plane velocity components can be obtained by integrating the in-plane acceleration components $\ddot{p}_{x}, \ddot{p}_{y}$, and $\alpha_{z}$ of the plate's motion.

Intuition about the qualitative properties of the field can often be gained by assuming that the part's dynamics are dominated by the portion of the cycle when $g_{\text {eff }}$ (and thus the transient acceleration of the part) is largest. This is discussed below for sinusoidal plate motions.

\section{B. Sinusoidal Motion Primitives}

We now focus on the class of plate motions whose linear and angular acceleration components are sinusoidal with the same frequency, $f$ :

$$
\begin{array}{ll}
\ddot{p}_{x}=A_{x} \sin \left(2 \pi f t+\phi_{x}\right) & \alpha_{x}=A_{\theta} \sin \left(2 \pi f t+\phi_{\theta}\right) \\
\ddot{p}_{y}=A_{y} \sin \left(2 \pi f t+\phi_{y}\right) & \alpha_{y}=A_{\varphi} \sin \left(2 \pi f t+\phi_{\varphi}\right) \\
\ddot{p}_{z}=A_{z} \sin \left(2 \pi f t+\phi_{z}\right) & \alpha_{z}=A_{\psi} \sin \left(2 \pi f t+\phi_{\psi}\right) .
\end{array}
$$

This 11-dimensional space of plate motions is parameterized by the six amplitudes and five phases (one phase is chosen to be zero without loss of generality). We refer to plate motions of this form as sinusoidal motion primitives.

To qualitatively estimate the transient accelerations associated with sinusoidal motion primitives we assume that the frictional force during the instant in the cycle when $g_{\text {eff }}$ is a maximum dominates the overall part dynamics. Thus, the average magnitude of a during the transient period is roughly proportional to the maximum value of $g_{\text {eff }}$. Similarly, the average direction of a during the transient period roughly corresponds to the direction of $\dot{\mathbf{q}}$ at the instant when $g_{\text {eff }}$ is a maximum.

As an example, consider the point $\mathbf{r}_{x y}=(0.06,0)^{T} \mathrm{~m}$ on a plate undergoing the motion given in Figure $5(\mathrm{k})$ with period $T=0.03 \mathrm{~s}$. The circular trajectory of the plate's velocity $\mathbf{v}^{*}$ is depicted in Figure 4(a). In Figure 4(c) we see that $g_{\text {eff }}$ is a maximum at $t=\frac{3}{4} T$. By examining the $x$ and $y$ velocities 
of the plate at this time we expect the asymptotic velocity to have a negative $y$-component and no $x$-component. This is very nearly true-the asymptotic trajectory to which the parts are converging in Figure 4(a) has an average $y$-velocity that is negative and an average $x$-velocity that is very close to zero, although slightly negative.

\section{ASYMPTOTIC VELOCITY FIELDS ARISING FROM SinUSOIDAL MOTION PRIMITIVES}

In this section we look at two classes of sinusoidal motion primitives. For these classes, we use estimates of transient accelerations to predict the qualitative behavior of the asymptotic velocity fields. Numerically calculated versions of these fields, as well as several other fields, are shown in Figure 5. Below each field is the sinusoidal motion primitive from which the field is generated and the approximate form of the asymptotic velocity. Six seconds of simulated motion of a point part starting from rest is overlaid on each field. The simulation and the fields are based on the full dynamic system model given in Section III (using the simplified system model in Section IV to generate the fields gives visually indistinguishable results). After a brief transient period, agreement between the simulated motion and the asymptotic velocity field is very good in all cases.

\section{A. Combining In-Plane Translation with Out-of-Plane Rota- tion: Nodal Line Fields}

The class of nodal line sinusoidal motion primitives combine the in-plane acceleration components $\ddot{p}_{x}$ and $\ddot{p}_{y}$ with the out-of-plane acceleration components $\alpha_{x}$ and $\alpha_{y}$ as follows:

$$
\begin{array}{ll}
\ddot{p}_{x}=A_{x} \sin (2 \pi f t) & \alpha_{x}=A_{\theta} \sin (2 \pi f t+\phi) \\
\ddot{p}_{y}=A_{y} \sin (2 \pi f t) & \alpha_{y}=A_{\varphi} \sin (2 \pi f t+\phi) .
\end{array}
$$

From (19) and (20), the effective gravity and relative velocity vector can be written as

$$
\begin{aligned}
g_{\mathrm{eff}} & \approx A_{\theta} \sin (2 \pi f t+\phi) y-A_{\varphi} \sin (2 \pi f t+\phi) x+g \\
\dot{\mathbf{q}} & \approx\left[\begin{array}{c}
-\dot{p}_{x} \\
-\dot{p}_{y} \\
0
\end{array}\right]=\left[\begin{array}{c}
\frac{A_{x}}{2 \pi f} \cos (2 \pi f t) \\
\frac{A_{y}}{2 \pi f} \cos (2 \pi f t) \\
0
\end{array}\right] .
\end{aligned}
$$

We note that $g_{\text {eff }}$ is position-dependent; its maximum value increases with distance from the line through the origin in the direction of $\left(A_{\theta}, A_{\varphi}\right)$. We refer to this line as a nodal line. We also note that $\dot{\mathbf{q}}$ is position-independent and points in the direction of the vector $\left(A_{x}, A_{y}\right)$. It follows that during the transient period a part will accelerate in a direction corresponding to $\pm\left(A_{x}, A_{y}\right)$ with a magnitude that scales with its distance from the nodal line.

Let us examine the special case where $A_{y}=A_{\theta}=0$, implying that $\dot{\mathbf{q}}$ is always aligned with the $x$-axis and that the maximum value of $g_{\text {eff }}$ increases with distance from the $y$-axis. Thus, we expect the magnitude of the transient acceleration to increase with distance from the $y$-axis. Further, whenever $g_{\mathrm{eff}}$ is a maximum on one side of the $y$-axis it is a minimum on the other side. This introduces an asymmetry that causes the direction of the part's transient acceleration to differ on opposite sides of the $y$-axis. Depending on the phase $\phi$ the part will accelerate toward or away from the nodal line.

For example, if $\phi=\frac{3}{2} \pi, g_{\text {eff }}$ and $\dot{\mathbf{q}}$ are out of phase with each other for positions satisfying $x<0$ (i.e., for parts with negative $x$-positions, the plate moves in the positive $x$-direction during the instant in the cycle when $g_{\text {eff }}$ is a maximum). On the other hand, the plate moves in the negative $x$-direction during the instant in the cycle when $g_{\text {eff }}$ is a maximum for parts satisfying $x>0$. It follows that during the transient period parts with $x<0$ tend to get accelerated in the positive $x$-direction whereas parts with $x>0$ tend to get accelerated in the negative $x$-direction. As illustrated in Figure 5(c), the asymptotic velocity field for this case corresponds to a squeeze field converging on the $y$-axis. We refer to this as a LineSink field.

In general, the class of nodal line sinusoidal motion primitives create a nodal line of zero velocity in the direction of the rotation axis (i.e., the direction of the vector $\left.\left(A_{\theta}, A_{\varphi}\right)\right)$. The value of $\phi$ determines whether the nodal line is attractive or repulsive. As illustrated in Figure 5(e)-(g), $A_{x}, A_{y}, A_{\theta}$, $A_{\varphi}$, and $\phi$ can be chosen to create fields such as SkewSink, Skewsource, and shear.

\section{B. Combining In-Plane Translation with Out-of-Plane Rota- tion: Nodal Fields}

The class of nodal sinusoidal motion primitives combine the in-plane acceleration components $\ddot{p}_{x}$ and $\ddot{p}_{y}$ with the outof-plane acceleration components $\alpha_{x}$ and $\alpha_{y}$ as follows:

$$
\begin{array}{ll}
\ddot{p}_{x}=A_{x} \sin (2 \pi f t) & \alpha_{x}=A_{\theta} \sin (2 \pi f t+\phi) \\
\ddot{p}_{y}=A_{y} \sin (2 \pi f t+\pi / 2) & \alpha_{y}=A_{\varphi} \sin (2 \pi f t+\pi / 2+\phi) .
\end{array}
$$

From (19) and (20), the effective gravity and relative velocity vector can be written as

$$
\begin{gathered}
g_{\text {eff }} \approx A_{\theta} \sin (2 \pi f t+\phi) y-A_{\varphi} \sin (2 \pi f t+\pi / 2+\phi) x+g \\
\dot{\mathbf{q}} \approx\left[\begin{array}{c}
-\dot{p}_{x} \\
-\dot{p}_{y} \\
0
\end{array}\right]=\left[\begin{array}{c}
\frac{A_{x}}{2 \pi f} \cos (2 \pi f t) \\
-\frac{A_{y}}{2 \pi f} \sin (2 \pi f t) \\
0
\end{array}\right] .
\end{gathered}
$$

The relative velocity $\dot{\mathbf{q}}$ is position-independent and rotates at a constant rate with a constant magnitude. This implies that the part can potentially accelerate in any direction at any location during the transient period. However, we can rule out many possibilities by examining $g_{\text {eff }}$. In particular, we expect the transient acceleration to be an odd function of position because when $g_{\text {eff }}(x, y, t)$ is a maximum $g_{\text {eff }}(-x,-y, t)$ is a minimum. Further, the magnitude of the transient acceleration should increase with distance from the origin because the maximum value of $g_{\text {eff }}$ increases in this manner.

In general, nodal sinusoidal motion primitives create fields with a node of zero velocity at the origin of the plate. The value of $\phi$ determines whether the node is attractive or repulsive as well as whether the field is oriented clockwise or counterclockwise. The values of $A_{x}, A_{y}, A_{\theta}$, and $A_{\varphi}$ determine the strength, orientation, and eccentricity of the 
(a) Trans

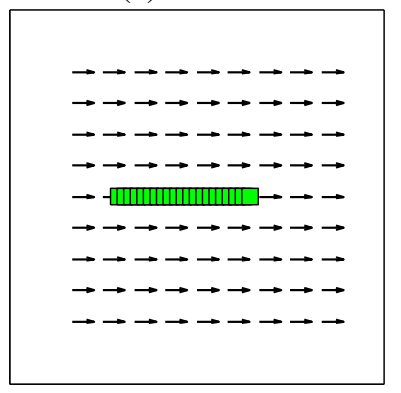

$\ddot{p}_{x}=10 \sin (66 \pi t)$

$\ddot{p}_{z}=5 \sin \left(66 \pi t+\frac{3}{2} \pi\right)$

$\mathbf{v}_{\mathrm{a}}(x, y) \approx(0.015,0)$

(e) SkewSink

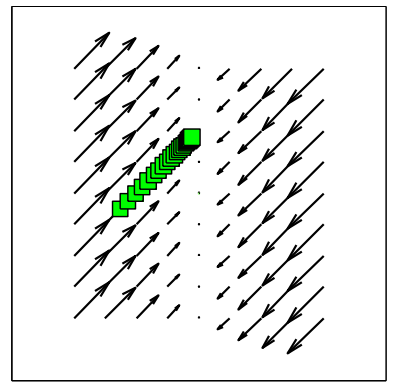

$\ddot{p}_{x}=10 \sin (66 \pi t)$

$\ddot{p}_{y}=10 \sin (66 \pi t)$

$\alpha_{y}=100 \sin \left(66 \pi t+\frac{3}{2} \pi\right)$

$\mathbf{v}_{\mathrm{a}}(x, y) \approx(-0.3 x,-0.3 x)$

(i) Sink

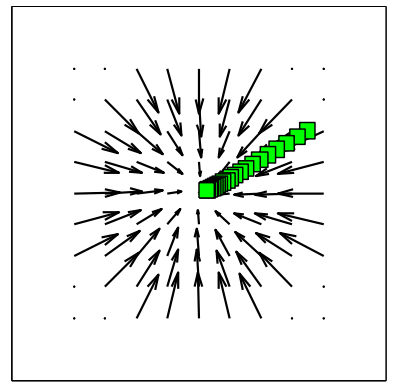

$\ddot{p}_{x}=10 \sin (66 \pi t)$

$\ddot{p}_{y}=10 \sin \left(66 \pi t+\frac{1}{2} \pi\right)$

$\alpha_{x}=100 \sin \left(66 \pi t+\frac{75}{64} \pi\right)$

$\alpha_{y}=100 \sin \left(66 \pi t+\frac{1}{2} \pi+\frac{75}{64} \pi\right)$

$\mathbf{v}_{\mathbf{a}}(x, y) \approx(-0.35 x,-0.35 y)$ (b) Circle

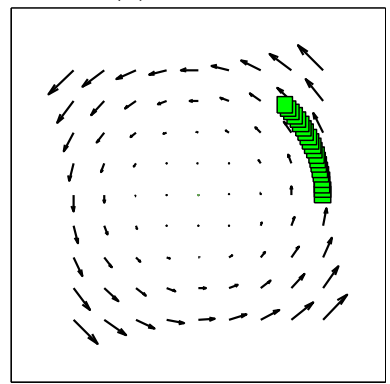

$\alpha_{z}=100 \sin (66 \pi t)$

$\ddot{p}_{z}=8 \sin \left(66 \pi t+\frac{3}{2} \pi\right)$

$\mathbf{v}_{\mathrm{a}}(x, y) \approx(-0.15 y, 0.15 x)$

(f) SkewSource

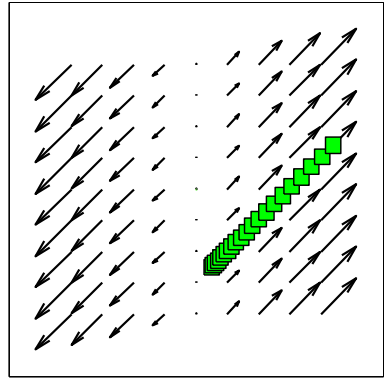

$\ddot{p}_{x}=10 \sin (66 \pi t)$

$\ddot{p}_{y}=10 \sin (66 \pi t)$

$\alpha_{y}=100 \sin \left(66 \pi t+\frac{1}{2} \pi\right)$

$\mathbf{v}_{\mathrm{a}}(x, y) \approx(0.3 x, 0.3 x)$

(j) Source

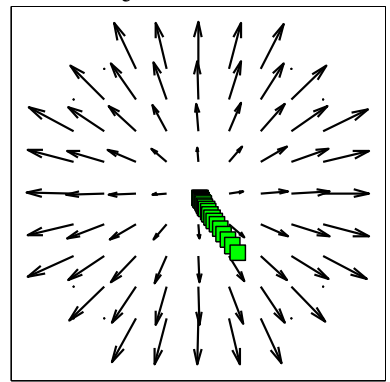

$\ddot{p}_{x}=10 \sin (66 \pi t)$

$\ddot{p}_{y}=10 \sin \left(66 \pi t+\frac{1}{2} \pi\right)$

$\alpha_{x}=100 \sin \left(66 \pi t+\frac{11}{64} \pi\right)$

$\alpha_{y}=100 \sin \left(66 \pi t+\frac{1}{2} \pi+\frac{11}{64} \pi\right)$

$\mathbf{v}_{\mathrm{a}}(x, y) \approx(0.35 x, 0.35 y)$ (c) LineSink

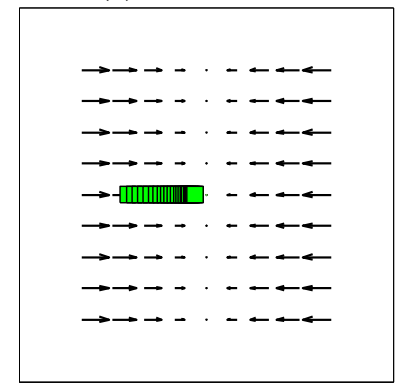

$\ddot{p}_{x}=10 \sin (66 \pi t)$

$\alpha_{y}=100 \sin \left(66 \pi t+\frac{3}{2} \pi\right)$

$\mathbf{v}_{\mathrm{a}}(x, y) \approx(-0.25 x, 0)$

(g) Shear

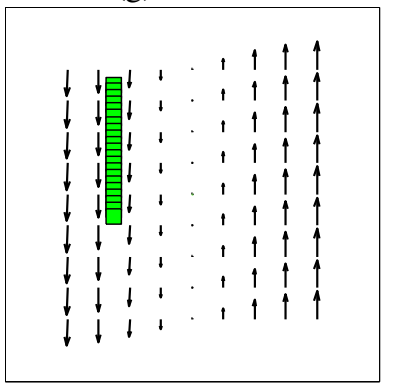

$\ddot{p}_{y}=10 \sin (66 \pi t)$

$\alpha_{y}=100 \sin \left(66 \pi t+\frac{3}{2} \pi\right)$

$\mathbf{v}_{\mathrm{a}}(x, y) \approx(0,0.25 x)$

(k) Whirlpool

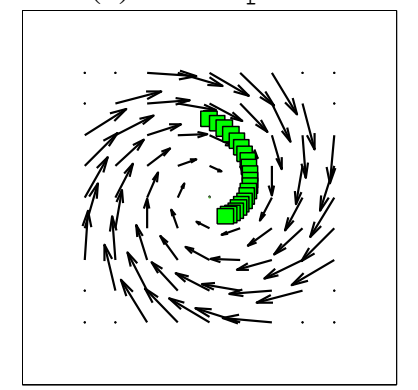

$\ddot{p}_{x}=10 \sin (66 \pi t)$

$\ddot{p}_{y}=10 \sin \left(66 \pi t+\frac{1}{2} \pi\right)$

$\alpha_{x}=100 \sin \left(66 \pi t+\frac{3}{2} \pi\right)$

$\alpha_{y}=100 \sin \left(66 \pi t+\frac{1}{2} \pi+\frac{3}{2} \pi\right)$

$$
\begin{gathered}
\mathbf{v}_{\mathrm{a}}(x, y) \approx \\
(-0.15 x+0.3 y,-0.3 x-0.15 y)
\end{gathered}
$$

(d) LineSource

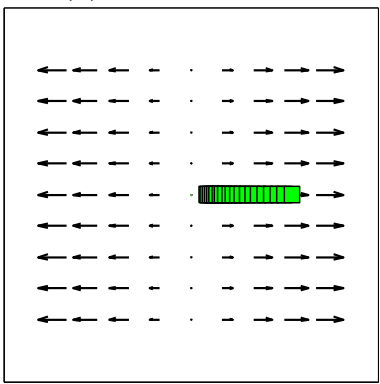

$\ddot{p}_{x}=10 \sin (66 \pi t)$

$\alpha_{y}=100 \sin \left(66 \pi t+\frac{1}{2} \pi\right)$

$\mathbf{v}_{\mathrm{a}}(x, y) \approx(0.25 x, 0)$ (h) DivCircle

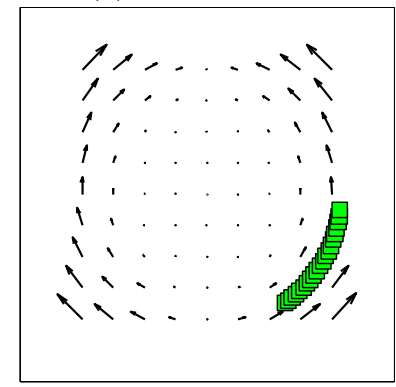

$\alpha_{z}=100 \sin (66 \pi t)$

$\alpha_{y}=10 \sin \left(66 \pi t+\frac{1}{2} \pi\right)$

$\mathbf{v}_{\mathrm{a}}(x, y) \approx\left(-2 x y, 2 x^{2}\right)$

Fig. 5. Numerically calculated asymptotic velocity fields based on the full dynamic model corresponding to sinusoidal motion primitives (when the fields are calculated with the simplified dynamic model the results are visually indistinguishable). The fields are calculated for a point part with $\mu_{k}=\mu_{s}=0.3$. Arrows are drawn in $2 \mathrm{~cm}$ increments. The arrows are missing in the corners of (i)-(l) because the part lost contact with the plate at those locations before reaching an asymptotic velocity. The sinusoidal motion primitive for each field is listed below it; linear and angular accelerations are in $\mathrm{m} / \mathrm{s}^{2}$ and $\mathrm{rad} / \mathrm{s}^{2}$ respectively. All acceleration components have a frequency of $33 \mathrm{~Hz}(T=0.03 \mathrm{~s})$. Below each sinusoidal motion primitive is an approximate form of the asymptotic velocity field given in units of $\mathrm{m} / \mathrm{s}$. Overlaid on each asymptotic velocity field is a six second (200 cycle) simulation of a point part starting from rest incorporating the full system dynamics. The position of the part is plotted every 0.3 seconds (every 10 cycles). 


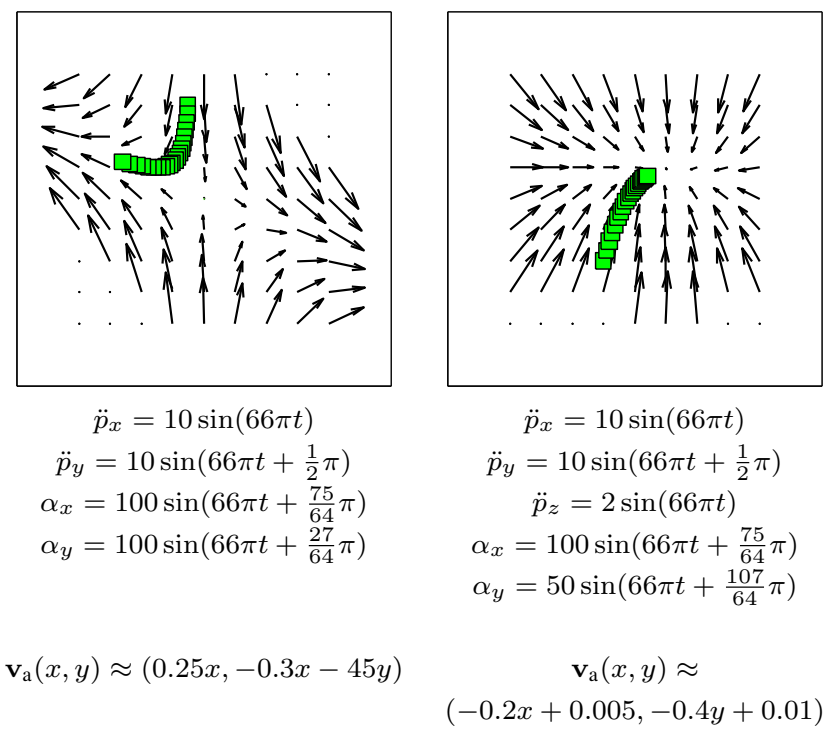

Fig. 6. Two asymptotic velocity fields with broken symmetries generated from sinusoidal motion primitives. The saddle on the left has nonorthogonal axes. The sink on the right biases motion in the $y$-direction and its node is shifted to the right and above the origin of the plate. Details about how the fields and simulated part motions were generated are given in the caption of Figure 5.

field. As illustrated in Figure 5(i)-(1), nodal fields include Sink, Source, Whirlpool, and Centrifuge.

\section{The Set of Obtainable Fields for Sinusoidal Plate Motion}

Based on the expressions for $\mathbf{v}_{a}$ given in Figure 5, we hypothesize that the class of sinusoidal motion primitives generate quadratic asymptotic velocity fields of the form

$$
\mathbf{v}_{\mathrm{a}}\left(\mathbf{r}_{x y}\right)=\mathbf{r}_{x y}^{T} \mathbf{A} \mathbf{r}_{x y}+\mathbf{B} \mathbf{r}_{x y}+\mathbf{c},
$$

where $\mathbf{A} \in \mathbb{R}^{2 \times 2 \times 2}, \mathbf{B} \in \mathbb{R}^{2 \times 2}$, and $\mathbf{c} \in \mathbb{R}^{2}$. The six combined elements of $\mathbf{B}$ and $\mathbf{c}$ can all be chosen independently, but there are constraints on $\mathbf{A}$ that are not yet fully understood.

This set of fields is a small subset of all possible asymptotic velocity fields obtainable with a six-DoF oscillating plate. Nonetheless, it is an interesting subset because it includes common fields with nonzero divergence. Figure 6 shows two asymptotic velocity fields that can be described by (21) that exhibit less symmetry than those in Figure 5.

\section{CONCLUSIONS}

We have presented a model that predicts a relationship between small-amplitude periodic motions of a rigid plate and position-dependent velocity fields for point parts in contact with the plate. The model predicts the existence of velocity fields with nonzero divergence; the accompanying videos of our six-DoF prototype device qualitatively verify that these fields can indeed be generated in practice. Numerical simulations in this paper support the theoretical validity of the model.

There are four broad areas that we see as fruitful for future work. The first is to gain more insight about the scope of obtainable fields for more general periodic plate motions. The second is to develop a comprehensive understanding of how to map a desired field to its associated periodic plate motion(s).
The third is to better understand how the coefficient of friction and the motion of the plate affect the rate of convergence to the asymptotic velocity. The fourth is to extend the work presented in this paper to handle parts with planar extent. These results will allow us to address a variety of applications including planar parts sorting, feeding, and assembly.

\section{REFERENCES}

[1] K.-F. Böhringer, B. R. Donald, L. E. Kavraki, and F. Lamiraux, "A single universal force field can uniquely orient non-symmetric parts," in International Symposium on Robotics Research. Springer, 1999, pp. 395-402.

[2] M. Coutinho and P. Will, "A general theory for positioning and orienting 2 d polygonal or curved parts using intelligent motion surfaces," in IEEE International Conference on Robotics and Automation, 1998, pp. 856862

[3] F. Lamiraux and L. Kavraki, "Positioning symmetric and non-symmetric parts using radial and constant force fields," in Workshop on the Algorithmic Foundations of Robotics, 2000.

[4] A. Sudsang, "Sensorless sorting of two parts in the plane using programmable force fields," in IEEE International Conference on Intelligent Robots and Systems, 2002, pp. 1784-1789.

[5] K.-F. Böhringer, B. Donald, and N. MacDonald, "Sensorless manipulation using massively parallel microfabricated actuator arrays," in IEEE International Conference on Robotics and Automation, 1994.

[6] K.-F. Böhringer, K. Goldberg, M. Cohn, R. Howe, and A. Pisano, "Parallel microassembly with electrostatic force fields," in IEEE International Conference on Robotics and Automation, 1998.

[7] S. Konoshi and H. Fujita, "A conveyance system using air flow based on the concept of distributed micro motion systems," Journal of Microelectromechanical Systems, vol. 3, no. 2, pp. 54-58, 1994.

[8] J. Luntz, W. Messner, and H. Choset, "Velocity field design for parcel manipulation on the modular distributed manipulator system," in IEEE International Conference on Robotics and Automation, 1999.

[9] J. Luntz and H. Moon, "Distributed manipulation with passive air flow," in IEEE/RSJ International Conference on Intelligent Robots and Systems, 2001.

[10] P. U. Frei, M. Wiesendanger, R. Büchi, and L. Ruf, "Simultaneous planar transport of multiple objects on individual trajectories using friction forces," in Distributed Manipulation, K. F. Böhringer and H. Choset, Eds. Kluwer Academic Publishers, 2000, pp. 49-64.

[11] D. Reznik, E. Moshkovich, and J. Canny, "Building a universal planar manipulator," in Workshop on Distributed Manipulation at the International Conference on Robotics and Automation, 1999.

[12] D. Reznik and J. Canny, "A flat rigid plate is a universal planar manipulator," in IEEE International Conference on Robotics and Automation, 1998, pp. 1471-1477.

[13] — , "The Coulomb pump: a novel parts feeding method using a horizontally-vibrating surface," in IEEE International Conference on Robotics and Automation, 1998, pp. 869-874.

[14] D. Reznik, J. Canny, and K. Goldberg, "Analysis of part motion on a longitudinally vibrating plate," in IEEE/RSJ International Conference on Intelligent Robots and Systems, 1997, pp. 421-427.

[15] S. Okabe, Y. Kamiya, K. Tsujikado, and Y. Yokoyama, "Vibratory feeding by nonsinusoidal vibration-optimum wave form," ASME Journal of Vibration, Acoustics, Stress, and Reliability in Design, vol. 107, pp. 188-195, Apr. 1985.

[16] W. A. Morcos, "On the design of oscillating conveyers-case of simultaneous normal and longitudinal oscillations," ASME Journal of Engineering for Industry, vol. 92, no. 1, pp. 53-61, 1970.

[17] P. Umbanhowar and K. Lynch, "Optimal vibratory stick-slip transport," IEEE Transactions on Automation Science and Engineering, vol. 5, no. 3, 2008.

[18] D. Reznik and J. Canny, "C'mon part, do the local motion!" in IEEE International Conference on Robotics and Automation, 2001, pp. 22352242 .

[19] T. Vose, P. Umbanhowar, and K. Lynch, "Vibration-induced frictional force fields on a rigid plate," in IEEE International Conference on Robotics and Automation, 2007.

[20] _ , "Friction-induced lines of attraction and repulsion for parts sliding on an oscillated plate," IEEE Transactions on Automation Science and Engineering, to appear. 